\title{
Romarias no município de Juazeiro do Norte, Ceará: perfil da demanda por atendimento de saúde e sazonalidade de internações*
}

doi: $10.5123 / 51679-49742015000100005$

\section{Pilgrimages in Juazeiro do Norte, Ceará, Brazil: profile of demand for healthcare assistance and seasonality of hospitalizations}

Marina Pereira de Vasconcelos

Secretaria Municipal de Saúde, Departamento de Vigilância Sanitária, Juazeiro do Norte-CE, Brasil

Cynthia Braga

Fundação Instituto Oswaldo Cruz, Centro de Pesquisas Aggeu Magalhães, Recife-PE, Brasil

Giselle Campozana Gouveia

Fundação Instituto Oswaldo Cruz, Centro de Pesquisas Aggeu Magalhães, Recife-PE, Brasil

Wayner Vieira de Souza

Fundação Instituto Oswaldo Cruz, Centro de Pesquisas Aggeu Magalhães, Recife-PE, Brasil

\section{Resumo}

Objetivo: descrever o perfil de morbidade de romeiros e investigar a variação sazonal das internações durante período de romaria em Juazeiro do Norte, Ceará, Brasil. Métodos: estudo transversal com dados dos atendimentos na romaria de Finados, 2 de novembro de 2012, e desenho ecológico de séries temporais sobre internações de 2007 a 2012; foram calculados índices de sazonalidade (IS). Resultados: entre 377 romeiros atendidos, a maioria tinha idade superior a 55 anos (53,5\%) e procurou atendimento para aferir a pressão arterial $(71,1 \%)$; observou-se aumento das internações por doenças respiratórias em abril $\left(\mathrm{IS}=1,40 ; \mathrm{IC}_{95 \%} 1,13-1,67\right)$, maio $\left(\mathrm{IS}=1,21 ; \mathrm{IC}_{95 \%} 1,03-1,40\right)$ e junho $\left(\mathrm{IS}=1,30 ; \mathrm{IC}_{95 \%} 1,04-1,55\right)$, e por doenças circulatórias em junho (IS=1,23; $\left.\mathrm{IC}_{95 \%} 1,15-1,24\right)$, julho (IS=1,15; IC $\left.{ }_{95 \%} 1,06-1,24\right)$ e agosto $\left(I S=1,09 ; \mathrm{IC}_{95 \%}\right.$ 1,06-1,11). Conclusão: o perfil de morbidade com predomínio de doenças do aparelho circulatório foi compatível com a faixa etária dos romeiros; não houve incremento das internações no período da romaria.

Palavras-chave: Morbidade; Acesso aos Serviços de Saúde; Distribuição Temporal; Epidemiologia Descritiva.

\begin{abstract}
Objective:to describe the morbidity pattern among pilgrims and investigate seasonal hospitalization variation in periods of pilgrimage in Juazeiro do Norte, Ceará, Brazil. Methods: a cross-sectional study with medical assistance data during the November 2nd 2012, All Soul's Day pilgrimage; and an ecological time series study to investigate hospitalization seasonality between 2007 and 2012; seasonal indexes (SI) were calculated. Results: among the 377 assisted pilgrims, most of them were more than 55 years old (53.5\%) and looked for medical assistance to measure blood pressure (71.1\%); seasonal hospitalization increase was observed for respiratory diseases in April (SI=1.40, 95\%CI 1.13-1.67), May (SI=1.21; 95\%CI 1.03-1.40) and June (IS=1.30; 95\%CI 1.04-1.55), and for circulatory diseases in June (SI=1.23; 95\%CI 1.16-1.31), July (SI=1.15; 95\%CI 1.06-1,24) and August (SI=1.09; 95\%CI 1.06-1.11). Conclusion: the morbidity pattern with prevalence of circulatory diseases was compatible with the age of pilgrims; there was no increase in hospitalizations in the period of pilgrimage.
\end{abstract}

Key words: Morbidity; Health Services Accessibility; Temporal Distribution; Epidemiology, Descriptive.

* Dissertação de mestrado profissional de Marina Pereira de Vasconcelos, intitulada 'Perfil de morbidade em serviço de prontoatendimento a romeiros e análise temporal das internações hospitalares nos períodos de romarias do município de Juazeiro do Norte-CE', defendida junto ao Programa de Pós-Graduação em Saúde Pública do Centro de Pesquisas Aggeu Magalhães/Fiocruz, em Pernambuco, Brasil, no ano de 2013.

Endereço para correspondência:

Cynthia Braga - Fundação Instituto Oswaldo Cruz, Centro de Pesquisas Aggeu Magalhães, Av. Professor Moraes Rego, s/n,

Campus da UFPE, Recife-PE, Brasil. CEP: 50670-420

E-mail:braga@cpqam.fiocruz.br 


\section{Introdução}

Eventos de massa, como peregrinações a lugares considerados sagrados, competições esportivas e concertos de música, estão se tornando, progressivamente, maiores e mais frequentes no mundo, e constituem um risco potencial de propagação de doenças infecciosas e de outros agravos à saúde da população, além de demandarem maior oferta de serviços de saúde. ${ }^{1}$

As peregrinações mais conhecidas no âmbito mundial e de maior importância no setor turístico religioso são as ocorridas nas cidades de Meca (Arábia Saudita), Jerusalém (Israel), Fátima, Lourdes (França) e Assis (Portugal), e no Vaticano. ${ }^{2}$ Destas, a maior peregrinação ocorre na cidade de Meca, em evento anual denominado Hajj, do qual participam mais de 2 milhões de muçulmanos oriundos de mais de 140 países. ${ }^{3}$

\section{Eventos de massa constituem} um desafio aos gestores de saúde por demandarem a criação de programas e a definição de políticas na área de Saúde Pública.

No Brasil, os principais eventos religiosos são o Círio de Nazaré, na cidade de Belém (PA), a festa da padroeira Nossa Senhora Aparecida, em Aparecida do Norte-SP, as festas de Iemanjá e do Nosso Senhor do Bonfim, em Salvador-BA, e a romaria de Padre Cícero, em Juazeiro do Norte, no estado do Ceará. ${ }^{4} \mathrm{~A}$ cidade de Aparecida do Norte-SP, onde se encontra 0 Santuário Nacional de Nossa Senhora Aparecida, um dos maiores templos católicos do mundo, recebe cerca de 10 milhões de visitantes anualmente. ${ }^{5}$

No Brasil, a despeito de, há muitos anos, serem realizados vários encontros de massa, religiosos e de outra natureza, em decorrência da realização de eventos de abrangência mundial como a Copa das Confederações em 2013, a Jornada Mundial da Juventude e a Copa do Mundo da Federação Internacional de Futebol (FIFA) em 2014, e os Jogos Olímpicos e Paraolímpicos, previstos para se realizarem em 2016, somente no mês junho de 2013 o Ministério da Saúde instituiu uma portaria regulamentando as diretrizes nacionais e as responsabilidades de esferas de gestão, no âmbito do Sistema Único de Saúde (SUS), para o planejamento, execução e avaliação das ações de vigilância e assistência à saúde em grandes e maciços eventos. ${ }^{6}$

Eventos de massa constituem um desafio aos gestores de saúde por demandarem a criação de programas e a definição de políticas na área de Saúde Pública, com o objetivo de prevenir a importação de doenças, promover e proteger a saúde dos visitantes e da população local. ${ }^{7,8}$ A elevada concentração de pessoas em uma área geográfica restrita, aliada às más condições de higiene pessoal e nutricional, à falta de higiene na preparação dos alimentos e à inadequada gestão de resíduos, além da situação de saneamento insatisfatória segundo cada localidade, aumentam os riscos de disseminação de doenças infecciosas e contribuem para a elevação das taxas de morbidade e mortalidade por doenças transmissíveis. ${ }^{1,8}$ Por sua vez, o retorno dos peregrinos infectados a seus locais de origem favorece a disseminação de agentes infecciosos para outras regiões e até países. ${ }^{8}$ Ademais, a participação nesses eventos expõe os viajantes a outros problemas no âmbito da saúde, como o agravamento de doenças crônico-degenerativas pré-existentes, traumas e mortes decorrentes de atos de violência e ataques terroristas. ${ }^{9,10}$

Entre os diversos locais de visitação por motivação religiosa no Brasil, a cidade de Juazeiro do Norte-CE, foco das romarias de devotos de Padre Cícero, recebe, em média, até 500 mil visitantes durante as festas religiosas, ${ }^{11}$ a maioria deles em busca da cura para seus problemas de saúde, os quais, muitas vezes, demandam assistência dos serviços públicos, como consultas médicas, dispensação de medicamentos ou internações.

No país, existem poucas informações sobre o perfil de morbidade dos peregrinos que frequentam locais de visitação religiosa. 0 único estudo registrado na literatura foi conduzido por Guimarães, ${ }^{12}$ que investigou a ocorrência de endemias rurais em peregrinos da romaria de Bom Jesus da Lapa, na Bahia, tendo identificado casos de malária, leishmaniose, varíola, micoses, febre tifoide, doença de Chagas, verminoses e doenças sexualmente transmissíveis (DST) como a sífilis, além de outros agravos. Outrossim, desconhece-se o impacto das romarias nos serviços de saúde do município em questão.

Este artigo tem por objetivos descrever o perfil de morbidade de romeiros atendidos em um posto de pronto atendimento e investigar a variação sazonal no 
número de internações em períodos correspondentes à realização das principais romarias no município de Juazeiro do Norte-CE.

\section{Métodos}

0 estudo incluiu um desenho transversal, que envolveu a coleta de dados primários para o levantamento da frequência e desenho do perfil da demanda por atendimentos de saúde dos peregrinos durante a romaria de Finados, em 2012. Adicionalmente, foi realizado um estudo ecológico, com dados do Sistema de Informações Hospitalares do Sistema Único de Saúde (SIH/SUS) relativos ao município de Juazeiro do Norte-CE, no período de 2007 a 2012, com o propósito de investigar a existência de variação sazonal no número de internações por causas específicas nos meses correspondentes à realização das principais romarias no município.

Juazeiro do Norte-CE se situa na Região Metropolitana do Cariri, estado do Ceará, apresenta uma área total de $248,83 \mathrm{~km}^{2}$ e possui uma população de cerca de 250 mil habitantes. ${ }^{13}$ À época do estudo, a rede de serviços de saúde do município contava com mais de 300 estabelecimentos de saúde, os quais ofertavam serviços de atenção primária e assistência de média e de alta complexidade. 0 município possuía quatro unidades hospitalares públicas: Hospital Regional do Cariri, Hospital Tasso Ribeiro Jereissati, Hospital São Lucas e Hospital Infantil Maria Amélia. Estes dois últimos serviços, responsáveis pelo atendimento da população pediátrica. 0 Hospital Regional do Cariri, o maior de todos, é referência de atendimento para 44 municípios da macrorregião do Cariri.

Anualmente, são realizadas diversas romarias no município, sendo a romaria das Candeias (29 de janeiro a 2 de Fevereiro), a de Nossa Senhora das Dores (15 de setembro) e a romaria de Finados (29 de outubro a 2 de novembro) as mais importantes em número de visitantes. Estimativas apontam o afluxo de cerca de 600 mil romeiros durante a romaria de Finados, a maior romaria do ano na cidade. ${ }^{11}$ Nos períodos das romarias mais importantes - das Candeias, de Nossa Senhora das Dores e de Finados -, são instalados dois postos de pronto atendimento, gerenciados pela Secretaria Municipal de Saúde, que funcionam em áreas próximas aos locais de visitação e ofertam serviços de atenção primária.
A coleta de dados para o estudo transversal foi realizada no período da romaria de Finados, de 29 de outubro a 2 de novembro de 2012, na unidade de pronto atendimento que contava com atendimento médico, além de atendimento de enfermagem. Essa unidade funcionou no horário das 7 às $23 \mathrm{~h}$ nos três primeiros dias de romaria, e no turno da manhã (7 às $12 \mathrm{~h}$ ) no último dia de romaria.

Participaram do estudo, peregrinos de qualquer idade que procuraram atendimento nesse serviço. Inicialmente, os participantes foram atendidos por enfermeiros ou técnicos de enfermagem que levantavam os dados pessoais e clínicos (sinais e sintomas que motivaram a procura pelo atendimento), realizavam procedimentos de baixa complexidade, como aferição de pressão arterial, testes de glicemia ou verificação de temperatura quando necessário, e registravam as informações em um formulário padronizado e previamente testado. Casos mais graves eram atendidos pelo médico. As informações sobre os dados clínicos, impressão diagnóstica, conduta e desfecho (alta, administração de medicamentos e/ou observação, transferência ou óbito) foram registradas no formulário pelo profissional responsável do atendimento ou por um dos membros da equipe de pesquisadores.

As variáveis deste estudo foram a data e o turno do atendimento (manhã, tarde, noite), sexo (masculino, feminino), idade (em tercis: $<30,30-55$ e $\geq 56$ anos), local de residência (municipio e unidade da federação), motivo do atendimento (consulta, aferição de pressão arterial, glicemia, curativo, outro), sinais e sintomas apresentados (cefaleia, febre, sintomas do aparelho respiratório, sintomas do aparelho digestivo, dores em geral), diagnóstico (segundo os códigos da Classificação Estatística Internacional de Doenças e Problemas Relacionados à Saúde - CID 10 - presentes no diagnóstico fornecido pelo médico), medicamento administrado na unidade de saúde, medicamento dispensado para uso no domicílio e desfecho (alta, observação, transferência, óbito).

0 estudo ecológico foi realizado a partir de dados do SIH-SUS relativos ao período de janeiro de 2007 a novembro de 2012. As internações foram classificadas pelo mês e ano da internação e de acordo com os principais grupos de doenças que afetam os romeiros (doenças infecciosas e parasitárias, doenças endócrinas e metabólicas, doenças do aparelho circulatório, doenças do aparelho respiratório e causas externas), 
segundo dados da literatura, ${ }^{14-16}$ definidos com base na CID-10.

0 incremento sazonal no número de internações foi investigado nos meses de realização das principais romarias: das Candeias (janeiro e fevereiro), de Nossa Senhora das Dores (setembro) e de Finados (outubro e novembro).

A entrada e a análise dos dados do estudo transversal foram realizadas pelo programa Epi Info versão 3.5.4. Foi feita uma análise descritiva da distribuição de frequência (valores absolutos, percentuais, médias, medianas e desvio-padrão) dos atendimentos segundo as características sociodemográficas dos pacientes e do próprio atendimento. As diferenças na distribuição de frequência de sinais e sintomas, segundo sexo e idade, foram testadas utilizando-se o teste do qui-quadrado de Yates.

A análise dos dados do estudo ecológico foi realizada com o auxílo do programa Statistical Package for the Social Sciences (SPSS) versão 17.0. Entende-se por variações sazonais, a ocorrência de oscilações de curto prazo sempre em determinados períodos do ano. ${ }^{17}$ Para a investigação de sazonalidade, inicialmente, obteve-se a média anual do número de internações segundo grupo de causas, para cada ano da série de 2007 a 2012. Posteriormente, os índices de sazonalidade (IS) de cada mês foram obtidos pelo cálculo da razão entre o número de internações registradas em cada mês e a média de internações ocorridas no respectivo ano. Por fim, foram calculadas as médias dos IS de cada mês para o período (2007-2012) e seus respectivos intervalos de confiança de $95 \%\left(\mathrm{IC}_{95 \%}\right.$ ). Médias de IS maiores que 1 , cujos IC $_{95 \%}$ não incluíssem a unidade, foram interpretadas como incremento sazonal estatisticamente significativo. Adotou-se um nível de significância de 5\%.

A pesquisa foi aprovada pelo Comitê de Ética do Centro de Pesquisas Aggeu Magalhães, da Fundação Instituto Oswaldo Cruz (Fiocruz), mediante o Parecer $n^{0}$ 27/2012 - registro do Certificado de Apresentação para Apreciação Ética (CAAE) $n^{0}$ 04676112.8.0000.5190.

\section{Resultados}

Um total de 377 peregrinos foram atendidos na unidade de pronto atendimento estudada, sem recusas. A idade da população variou de 0 a 86 anos, com média de 52,8 (desvio-padrão: 19,6 anos) e mediana de 57 anos. Houve maior frequência de atendimento a pessoas do sexo feminino $(64,5 \%)$, a maioria proveniente do estado de Pernambuco (52,9\%), seguido pelo estado de Sergipe $(25,9 \%)$. Apenas 7,8\% eram provenientes do estado do Ceará (Tabela 1).

\section{Tabela 1 - Características demográficas dos pacientes atendidos no posto de pronto-atendimento $(n=377)$ durante 0 período de romaria no município de Juazeiro do Norte, Ceará, outubro e novembro de 2012}

\begin{tabular}{|c|c|c|}
\hline Características & $\mathbf{N}$ & $\%$ \\
\hline \multicolumn{3}{|l|}{ Sexo } \\
\hline Masculino & 134 & 35,5 \\
\hline Feminino & 343 & 64,5 \\
\hline \multicolumn{3}{|l|}{ Grupo etário (em anos) ${ }^{a}$} \\
\hline$<30$ & 56 & 14,9 \\
\hline $30-55$ & 119 & 31,6 \\
\hline$\geq 56$ & 201 & 53,5 \\
\hline \multicolumn{3}{|l|}{ Reside em Juazeiro-CE } \\
\hline Sim & 28 & 7,4 \\
\hline Não & 349 & 92,6 \\
\hline \multicolumn{3}{|l|}{ Estado de procedênciab ${ }^{b}$} \\
\hline Pernambuco & 198 & 52,9 \\
\hline Sergipe & 97 & 25,9 \\
\hline Ceará & 29 & 7,8 \\
\hline Alagoas & 25 & 6,7 \\
\hline Outros estados da região Nordeste & 22 & 5,9 \\
\hline Estados da região Sudeste & 3 & 0,8 \\
\hline
\end{tabular}

a) 1 registro sem informação

b) 3 registros sem informação 
A maior parte dos atendimentos foi realizada entre 0 segundo e o quarto dias de romaria $(77,0 \%)$ e no turno da manhã (46,4\%). A maioria da população atendida procurou o serviço para aferição da pressão arterial $(71,1 \%)$ e atendimento médico $(20,7 \%)$. Do total de atendidos, 18,3\% necessitaram ser medicados no serviço e 8,8\% receberam medicação para tratamento domiciliar. Quanto ao desfecho, 90,2\% dos pacientes receberam alta após o atendimento, 7,4\% permaneceram em observação e 2,4\% foram transferidos para outro serviço de saúde (Tabela 2).

Entre os 163 romeiros atendidos por profissionais de saúde (médicos ou enfermeiros), 82 (50,3\%) eram portadores de doenças do aparelho circulatório, $22(13,5 \%)$ de doenças endócrinas, nutricionais e metabólicas, 15 (9,2\%) de doenças do aparelho respiratório, $11(6,7 \%)$ de doenças infecciosas e parasitárias e $33(20,3 \%)$ procuraram atendimento por outras causas (doenças de pele e do tecido subcutâneo, queimadura e traumas). Dois casos de varicela foram diagnosticados em menores de 12 anos de idade (dados não apresentados na tabela).

Dos 88 pacientes atendidos que referiram algum sinal ou sintoma, 34 queixavam-se de cefaleia, 31 apresentavam sintomas do aparelho digestivo, 28 tinham queixas respiratórias, 23 foram diagnosticados com febre e 19 queixavam-se de dores em geral (membros e corpo). A Tabela 3 apresenta a análise comparativa dos principais sinais e sintomas observados, segundo grupo etário e sexo. Houve maior frequência de relato de febre $(\mathrm{p}<0,001)$ e de sintomas do aparelho digestivo $(\mathrm{p}=0,040)$ no grupo etário mais jovem ( $<30$ anos), quando comparado aos grupos de idade mais avançada. Não foram

\section{Tabela 2 - Características dos atendimentos no posto de pronto atendimento $(n=377)$ durante o período de romaria no município de Juazeiro do Norte, Ceará, outubro e novembro de 2012}

\begin{tabular}{|c|c|c|}
\hline Características & N & $\%$ \\
\hline \multicolumn{3}{|l|}{ Data do atendimento } \\
\hline $29 / 10$ & 64 & 16,9 \\
\hline $30 / 10$ & 90 & 23,9 \\
\hline $31 / 10$ & 90 & 23,9 \\
\hline $1 / 11$ & 110 & 29,2 \\
\hline $2 / 11$ & 23 & 6,1 \\
\hline \multicolumn{3}{|l|}{ Turno } \\
\hline Manhã & 175 & 46,4 \\
\hline Tarde & 130 & 34,5 \\
\hline Noite & 72 & 19,1 \\
\hline \multicolumn{3}{|l|}{ Motivo da procura por atendimento } \\
\hline Atendimento médico & 78 & 20,7 \\
\hline Aferição de pressão arterial (PA) & 268 & 71,1 \\
\hline Teste de glicemia e aferição de PA & 13 & 0,8 \\
\hline Curativo & 5 & 1,3 \\
\hline Outro & 13 & 3,4 \\
\hline \multicolumn{3}{|l|}{ Administração de medicamento } \\
\hline Sim & 69 & 18,3 \\
\hline Não & 308 & 81,7 \\
\hline \multicolumn{3}{|l|}{ Dispensação de medicação } \\
\hline Sim & 33 & 8,8 \\
\hline Não & 344 & 91,2 \\
\hline \multicolumn{3}{|l|}{ Desfecho do atendimento } \\
\hline Alta & 340 & 90,2 \\
\hline Observação & 28 & 7,4 \\
\hline Transferência & 9 & 2,4 \\
\hline
\end{tabular}


Tabela 3 - Sinais e sintomas relatados pelos pacientes atendidos no posto de pronto atendimento ( $n=88$, segundo grupo etário e sexo, durante período de romaria no município de Juazeiro do Norte, Ceará, outubro e novembro, 2012

\begin{tabular}{|c|c|c|c|c|c|c|c|}
\hline \multirow[b]{2}{*}{ Sinais e sintomas } & \multicolumn{3}{|c|}{ Grupo etário (em anos) } & \multirow[b]{2}{*}{$\begin{array}{c}- \text { Valor de } \\
p^{a}\end{array}$} & \multicolumn{2}{|c|}{ Sexo } & \multirow{2}{*}{$\begin{array}{c}\text { Valor de } \\
\qquad \mathbf{p}^{\mathrm{a}}\end{array}$} \\
\hline & $\begin{array}{c}<30 \\
(n=29)\end{array}$ & $\begin{array}{c}30-55 \\
(n=29)\end{array}$ & $\begin{array}{c}>55 \\
(n=30)\end{array}$ & & $\begin{array}{c}\text { Masculino } \\
(n=30)\end{array}$ & $\begin{array}{c}\begin{array}{c}\text { Feminino } \\
(n=58)\end{array} \\
\end{array}$ & \\
\hline Febre & 14 & 4 & 4 & 0,000 & 11 & 11 & 0,119 \\
\hline Aparelho respiratório (dispneia/tosse/otalgia/odinofagia) & 10 & 9 & 7 & 0,629 & 9 & 17 & 0,934 \\
\hline Aparelho digestivo (dor abdominal, náuseas, diarreia e vômitos) & 15 & 11 & 6 & 0,040 & 10 & 22 & 0,848 \\
\hline Dores em geral (membros, corpo, dentes) & 7 & 11 & 7 & 0,380 & 9 & 16 & 0,812 \\
\hline Outros & 4 & 1 & 2 & 0,329 & 3 & 6 & 0,959 \\
\hline
\end{tabular}

a) Teste do qui-quadrado de Yates

observadas diferenças estatisticamente significativas segundo o sexo, na frequência dos sinais e sintomas relatados.

Quanto à administração de medicamentos, 69 $(18,3 \%)$ pacientes foram medicados na unidade de pronto atendimento,os medicamentos mais frequentemente administrados foram hipoglicemiante injetável (insulina regular), analgésicos e antipiréticos, e medicação anti-hipertensiva; $33(8,7 \%)$ pacientes receberam medicamentos para uso no domicílio: analgésicos e antipiréticos, antialérgicos e antibióticos foram os mais dispensados.

Observou-se incremento sazonal no número de internações por doenças do aparelho respiratório, nos meses de abril (IS=1,40; $\left.\mathrm{IC}_{95 \%}: 1,13-1,67\right)$, maio (IS=1,21; $\left.\mathrm{IC}_{95 \%}: 1,03-1,40\right)$ e junho (IS=1,30; $\left.\mathrm{IC}_{95 \%}: 1,04-1,55\right)$. Constatou-se, igualmente, incremento das internações por doenças do aparelho circulatório nos meses de junho (IS=1,23; $\mathrm{IC}_{95 \%}: 1,16$ $1,32)$, julho ( $\left.\mathrm{IS}=1,15 ; \mathrm{IC}_{95 \%}: 1,06-1,24\right)$ e agosto $\left(\mathrm{IS}=1,09 ; \mathrm{IC}_{95 \%}: 1,02-1,15\right)$. Nenhum incremento sazonal - estatisticamente significativo - foi observado nas internações pelos demais grupos de doenças (doenças endócrinas e metabólicas, doenças infecciosas e parasitárias). As figuras 1 e 2 mostram a variação mensal dos IS para as internações por doenças do aparelho respiratório e do aparelho cardiocirculatório, respectivamente.



Figura 1 - Índices de sazonalidade (médias e intervalo de confiança de 95\%) do número de internações por doenças do aparelho respiratório no município de Juazeiro do Norte, Ceará, 2007 a 2012 


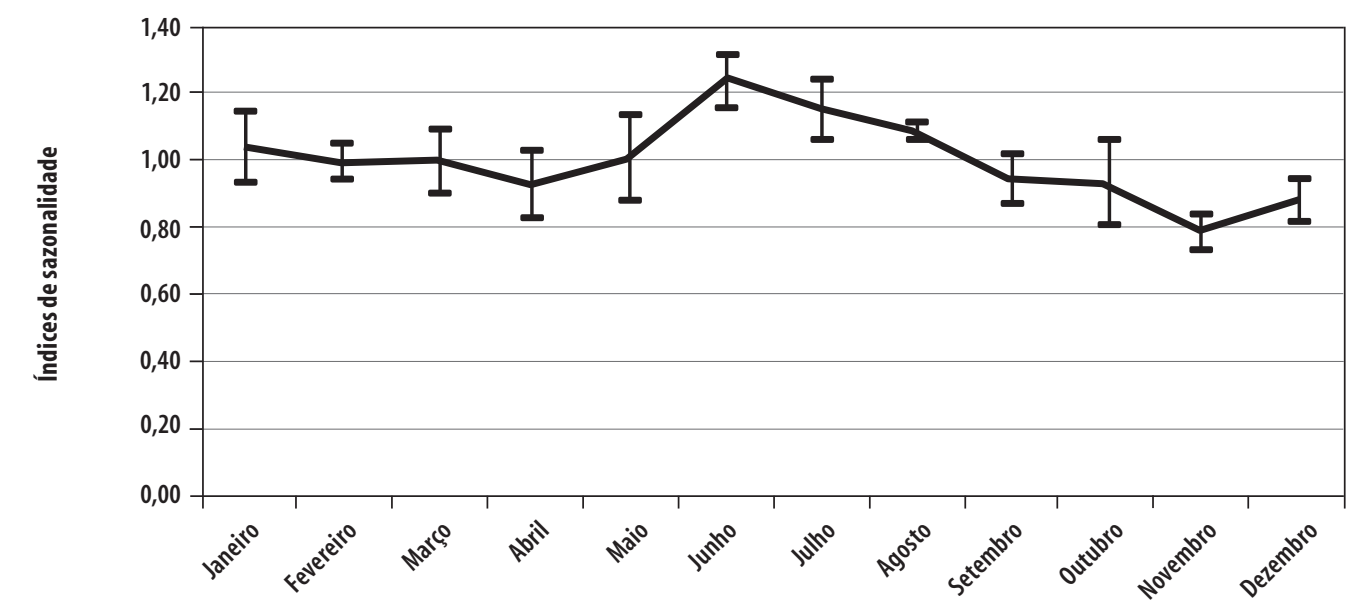

Figura 2 - Índices de sazonalidade (médias e intervalo de confiança de 95\%) do número de internações por doenças do aparelho cardiocirculatório no município de Juazeiro do Norte, Ceará, 2007 a 2012

\section{Discussão}

Este é o primeiro estudo a investigar o perfil de morbidade em populações de romeiros, em um dos principais sítios de peregrinação religiosa no Brasil. 0 levantamento mostrou que mais de $70 \%$ da população atendida procurou o posto de pronto atendimento para a realização de procedimentos simples, como aferição de pressão arterial e de glicemia, e que a maioria recebeu alta após o atendimento. Não foi observado incremento do número de internações nas unidades hospitalares públicas locais durante os períodos das principais romarias.

Os principais sinais e sintomas, motivadores da procura por atendimento, e o perfil de morbidade da população assistida foram semelhantes aos encontrados em outros sítios de peregrinação no mundo. ${ }^{9,14,15}$

A maior parte dos romeiros que procurou atendimento procedia de outros estados, principalmente de Pernambuco, situado na divisa com o estado do Ceará. Este dado chama a atenção para os movimentos populacionais ocasionados por eventos de massa e seus riscos para a propagação de doenças transmissíveis. ${ }^{1,8}$ Assim, a integração das ações de vigilância epidemiológica em fronteiras geográficas próximas a sítios de realização de eventos de massa, como as romarias, mostra-se importante para a rápi- da detecção de doenças infecciosas, particularmente da importação de doenças transmissíveis emergentes de alcance global. Tais ações devem ser executadas antes, durante e imediatamente após a ocorrência do evento. ${ }^{18}$

Neste estudo, de forma similar a levantamentos realizados em outros sítios de peregrinação no mundo, ${ }^{14-16}$ os principais sinais e sintomas que motivaram a procura dos romeiros por atendimento de saúde foram a cefaleia, seguida pelos distúrbios do aparelho digestivo, queixas respiratórias e febre, sem diferenças estatisticamente significantes entre os sexos. A febre e os sintomas do aparelho digestivo foram mais frequentes nos indivíduos mais jovens. A elevada ocorrência de cefaleia entre peregrinos é, possivelmente, decorrente do desconforto e precariedade dos meios de transporte utilizados no percurso, durante longos períodos, até o local da peregrinação, muitas vezes sobre a carroceria de caminhões adaptados, denominados 'paus-de-arara', sem proteção contra sol, poeira e chuva. A longa exposição dos romeiros ao sol durante as atividades religiosas, igualmente, parece contribuir para a elevada frequência desse sintoma.

Quantos aos distúrbios do aparelho digestivo, particularmente as gastroenterites, levantamentos realizados durante 0 Hajj, na cidade de Meca, ${ }^{19,20}$ demonstraram elevada frequência desse evento entre 
peregrinos, normalmente decorrentes da ingestão de alimentos elaborados sob precárias condições de higiene e mal conservados, além do consumo de água contaminada. As infecções gastrointestinais afetam não apenas a saúde dos viajantes como também representam risco de disseminação para locais fora do sítio de visitação, uma vez que enteroinfecções com período de incubação mais longo podem se manifestar mais tardiamente, após o retorno dos peregrinos a seus locais de origem. ${ }^{21}$

A elevada frequência de queixas respiratórias também vem-se observando em outros sítios de peregrinação no mundo: as infecções respiratórias agudas são uma importante causa de procura por atendimento e de mortes entre peregrinos. ${ }^{15,16,22} \mathrm{~A}$ maior ocorrência de infecções respiratórias nessa população tem sido atribuída à aglomeração de pessoas em uma área restrita, por um tempo relativamente longo e sob precárias condições de higiene, o que favorece a transmissão de patógenos respiratórios. ${ }^{23}$ Assim, as epidemias por patógenos respiratórios emergentes, como a pandemia pelo vírus influenza A (H1N1) ocorrida em 2009, são motivo de preocupação para as autoridades de saúde no mundo, em contextos de eventos de massa. ${ }^{24}$

No presente estudo, encontrou-se atendimentos de dois adolescentes diagnosticados com varicela. Isso destaca a importância dos serviços de saúde nos eventos de massa para o diagnóstico e o tratamento dos casos de doenças transmissíveis, assim como para a orientação dos indivíduos acometidos a absterem-se de frequentar locais de aglomeração, de modo a contribuir para evitar a propagação destas doenças.

A febre, sintoma que tem motivado o atendimento em sítios de peregrinação como Meca, ${ }^{14,25}$ é comumente associada às infecções agudas do trato respiratório que, frequentemente, afetam os peregrinos.

As doenças do aparelho circulatório, endócrinas e metabólicas foram os agravos diagnosticados com maior frequência entre os romeiros submetidos a exame clínico. Essa manifestação de morbidade é compatível com a faixa etária da população atendida, em sua maioria composta por indivíduos com idade superior a 40 anos, e condizente com o perfil epidemiológico da população brasileira. ${ }^{26}$ Estes resultados também corroboram os achados de levantamentos realizados em sítios de peregrinação de outros países. ${ }^{14,27}$
Cerca de $20 \%$ dos atendimentos demandaram a administração de medicamentos e sua dispensação a cerca de $10 \%$ dos pacientes atendidos, representando um custo adicional na assistência prestada aos romeiros pelo município. No Brasil, todavia, não há registros de levantamentos dos gastos públicos na prestação de serviços de saúde em eventos de massa. Assim, torna-se necessário o dimensionamento do impacto econômico de tais eventos no setor Saúde, em suas diferentes instâncias gestoras, além de ações de planejamento voltadas à prestação destes serviços e à alocação de recursos específicos para esse fim.

A análise de sazonalidade no número de internações registradas pelo SIH/SUS no município mostrou incremento sazonal significativo das internações por doenças do aparelho circulatório, nos meses de junho a agosto, e por doenças respiratórias, nos meses de abril a junho, não coincidindo com as datas de realização das principais romarias. Esses dados sugerem que as romarias, possivelmente, não têm levado a um aumento no número de internações na rede hospitalar do município. Com relação ao aumento do número de internações por doenças respiratórias, observado nos meses que correspondem ao período da estação chuvosa, é nesse período quando, normalmente, se observa aumento da ocorrência dessas afecções. ${ }^{28,29}$ Vale ressaltar que nesse mesmo período, acontecem as festas juninas, de grande importância e tradição, o maior evento festivo do município.

Os dados primários deste estudo são relativos às consultas realizadas em uma das três unidades de pronto atendimento existentes, durante a romaria de Finados, e não incluem os atendimentos ocorridos nos serviços de média e alta complexidade, cujos casos seriam potencialmente mais graves e com perfil de morbidade possivelmente distinto da população estudada. Com relação ao estudo ecológico, distorções decorrentes de erros diagnósticos e de preenchimento do formulário devem ser consideradas. Os resultados apresentados aqui, além de traçarem o perfil da demanda por atendimentos da população de romeiros em um dos principais sítios de peregrinação do Brasil, chamam a atenção para a necessidade do desenvolvimento de políticas de saúde voltadas à prevenção de agravos e assistência à saúde da população durante a realização de 
eventos de massa no Brasil. Torna-se igualmente necessário o fortalecimento das ações de planejamento em saúde, especialmente as de vigilância sanitária - e entre estas, particularmente, as de fiscalização do comércio de alimentos - e as de vigilância epidemiológica, visando a identificação precoce de doenças transmissíveis, a prevenção da ocorrência de surtos e epidemias, e da disseminação das doenças.

\section{Agradecimentos}

Agradecemos à Secretaria Municipal de Saúde de Juazeiro do Norte-CE, pelo apoio logístico.

\section{Referências}

1. Al-Tawfiq JA, Memish ZA. Mass gatherings and infectious diseases: prevention, detection, and control. Infect Dis Clin North Am. 2012 Sep;26(3):725-37.

2. Huff Post Religion. Religious pilgrimages: 14 of the world's most spiritual destinations [Internet]. 2012 [cited 2014 Mar 5]. Available from: http:// www.huffingtonpost.com/2012/06/03/religiouspilgrimages-spiritual-_n_1564664.html

3. Memish ZA, Venkatesh S, Ahmed QA. Travel epidemiology: the Saudi perspective. Int J Antimicrob Agents. 2003 Feb;21(2):96-101.

4. Teixeira MSG, Romão Jr MC. Turismo religioso: uma alternativa econômica para municípios do Seridó - RN [Internet]. 2013 [citado 2013 mai 18]. Disponível em: http://docente.ifrn.edu.br/marcosaraujo/ disciplinas/geografia-do-rio-grande-do-norte/ material-complementar/turismo-religioso-no-serido

5. Ministério do Turismo (BR). Tipos de turismo [Internet]. 2013 [citado 2013 mai 8]. Disponível em: http://www.brasil.gov.br/sobre/ turismo/tipos-deturismo/turismo-religioso

6. Brasil. Ministério da Saúde. Portaria $\mathrm{n}^{0} 1.139,10$ de junho de 2013 [Internet]. Define, no âmbito do Sistema único de Saúde (SUS), as responsabilidades das esferas de gestão e estabelece as Diretrizes Nacionais para Planejamento, Execução e Avaliação das Ações de Vigilância e Assistência à Saúde em Eventos de Massa. 2013 jun [citado 2013 out 6]. Disponível em: http://bvsms.saude.gov.br/bvs/ saudelegis/gm/2013/prt1139_10_06_2013.html

7. Polkinghorne BG, Massey PD, Durrheim DN, Byrnes T, MacIntyre CR. Prevention and surveillance of public

\section{Contribuição dos autores}

Vasconcelos MP e Braga C contribuíram com a concepção e delineamento do estudo, coleta dos dados, análise e redação, e revisão crítica do conteúdo intelectual do manuscrito.

Gouveia GC e Souza WV contribuíram com a análise, interpretação dos resultados e revisão crítica do conteúdo intelectual do manuscrito.

Todos os autores aprovaram a versão final do manuscrito e declaram serem responsáveis por todos os aspectos do trabalho, garantindo sua precisão e integridade.

health risks during extended mass gatherings in rural areas: the experience of the Tamworth Country Music Festival, Australia. Public Health. 2013 Jan;127(1):32-8.

8. Arguin PM, Marano N, Freedman DO. Globally mobile populations and the spread of emerging pathogens. Emerg Infect Dis. 2009 Nov;15(11):1713-4.

9. Steffen R, Bouchama A, Johansson A, Dvorak J, Isla $\mathrm{N}$, Smallwood C, et al. Non-communicable health risks during mass gatherings. Lancet Infect Dis. 2012 Feb;12(2):142-9.

10. Steffen R. Mass gatherings: health risks and preventive strategies. Ther Umsch. 2013 Jun;70(6):350-2.

11. Santos D. Milhares se unem para orar por seus mortos em Juazeiro do Norte [Internet]. 2013 nov [citado 2013 nov 8]. Disponível em: http:// www.opovo.com.br/app/opovo/radar/2013/11/02/ noticiasjornalradar,3157454/milhares-se-unempara-orar-por-seus-mortos-em-juazeiro-do-norte. shtml

12. Guimarães FN. Doenças encontradas nos romeiros de Bom Jesus da Lapa, Bahia. Mem Inst Oswaldo Cruz [Internet]. 1947 mar [citado 2012 abr 26];45(1): 211-51. Disponível em: http://www.scielo.br/pdf/ mioc/v45n1/tomo45\%28f1\%29_211-252.pdf

13. Instituto Brasileiro de Geografia e Estatística. Cidades@: Juazeiro do Norte-CE, censo demográfico 2010 [Internet]. Rio de janeiro: Instituto Brasileiro de Geografia e Estatística; 2010 [citado 2013 maio 8]. Disponível em: http://www.cidades.ibge.gov.br/xtras/ perfil.php?lang $=\&$ codmun $=230730$

14. Gautret P, Soula G, Delmont J, Parola P, Brouqui P. Common health hazards in French pilgrims during the 
Hajj of 2007: a prospective cohort study. J Travel Med. 2009 Nov-Dec;16(6):377-81.

15. Alzahrani AG, Choudhry AJ, Al Mazroa MA, Turkistani AH, Nouman GS, Memish ZA. Pattern of diseases among visitors to Mina health centers during the Hajj season, 1429 H (2008 G). J Infect Public Health. 2012 Mar;5(1):22-34.

16. Madani TA, Ghabrah TM, Al-Hedaithy MA, Alhazmi MA, Alazraqi TA, Albarrak AM, et al. Causes of hospitalization of pilgrims in the Hajj season of the Islamic year 1423 (2003). Ann Saudi Med. 2006 SepOct;26(5):346-51.

17. Latorre MRD0, Cardoso MRA. Análise de séries temporais em epidemiologia: introdução sobre os aspectos metodológicos. Rev Bras Epidemiol. 2001 nov;4(3):145-52.

18. Khan K, McNabb SJ, Memish ZA, Eckhardt R, Hu W, Kossowsky D, et al. Infectious disease surveillance and modelling across geographic frontiers and scientific specialties. Lancet Infect Dis. 2012 Mar;12(3):222-30.

19. Al-Ghamdi SM, Akbar OH, Qari YA, Fathaldin OA, Al-Rashed RS. Pattern of admission to hospitals during muslim pilgrimage (Hajj). Saudi Med J. 2003 Oct;24(10):1073-6.

20. Emamian MH, Mohammadi GM. An outbreak of gastroenteritis among Iranian pilgrims of Hajj during 2011. Iran Red Crescent Med J. 2013 Apr;5(4):317-19.

21. Karami M. Public health surveillance and Hajj pilgrimage as a mass gathering. Iran J Public Health. $2013 \mathrm{Jul} ; 42$ (7):791-2.

22. Pane M, Imari S, Alwi Q, Nyoman K, Cook AR, Samaan G. Causes of mortality for Indonesian Hajj pilgrims: comparison between routine death certificate and verbal autopsy findings. PLoS One. 2013 Aug;8(8):e73243.
23. Al-Tawfig JA, Smallwood CA, Arbuthnott KG, Malik MS, Barbeschi M, Memish ZA. Emerging respiratory and novel coronavirus 2012 infections and mass gathering. East Mediterr Health J. 2013;19 Suppl 13:48-54.

24. BalabanV, Stauffer WM, Hammad A, Afgarshe M, Abd-Alla M, Ahmed Q, et al. Protective practices and respiratory illness among US travelers to the 2009 Hajj. J Travel Med. 2012 May-Jun;19(3):163-8.

25. World Health Organization. Interim planning considerations for mass gatherings in the context of pandemic (H1N1) 2009 influenza [Internet]. Geneva: World Health Organization; 2009 [cited 2009 May 9]. Available from: http://www.who.int/csr/resources/ publications/swineflu/cp002_2009-0511_planning_ considerations_for_mass_gatherings.pdf

26. Duarte EC, Barreto SM. Transição demográfica e epidemiológica: a Epidemiologia e Serviços de Saúde revisita e atualiza o tema. Epidemiol Serv Saude. 2012 dez;21(4):529-32.

27. Khan NA, Ishag AM, Ahmad MS, El-Sayed FM, Bachal ZA, Abbas TG. Pattern of medical diseases and determinants of prognosis of hospitalization during 2005 Muslim pilgrimage Hajj in a tertiary care hospital. A prospective cohort study. Saudi Med J. 2006 Sep;27(9):1373-80.

28. Shek LPC, Lee BW. Epidemiology and seasonality of respiratory tract virus infections in the tropics. Paediatr Respir Rev. 2003 Jun;4(2):105-11.

29. Silva Jr JLR, Padilha TF, Rezende JE, Rabelo ECA, Ferreira ACG, Rabahi MF. Efeito da sazonalidade climática na ocorrência de sintomas respiratórios em uma cidade de clima tropical. J Bras Pneumol. 2011 nov-dez;37(6):759-67. 